\title{
Weight or metabolism: which deserve more attention in obesity?
}

\author{
Bing Zhu ${ }^{1,2}$, Aaron M. Gusdon ${ }^{3}$, Shen $Q u^{1,2,4}$ \\ ${ }^{1}$ Department of Endocrinology and Metabolism, Shanghai Tenth People's Hospital, School of Medicine, Tongji University, Shanghai 200072, China; \\ ${ }^{2}$ National Metabolic Management Center, Shanghai 200000, China; ${ }^{3}$ Division of Neurocritical Care, Department of Anesthesiology and Critical \\ Care Medicine, The Johns Hopkins Hospital, Baltimore, USA; ${ }^{4}$ Department of Endocrinology, School of Medicine, Nanjing Medical University, \\ Nanjing 210000, China \\ Correspondence to: Shen Qu, MD, PhD. Department of Endocrinology and Metabolism, Shanghai Tenth People's Hospital, School of Medicine, \\ Tongji University, Shanghai 200072, China. Email: qushencn@hotmail.com. \\ Provenance: This is an invited Editorial commissioned by the Section Editor Kaiping Zhang (AME College, AME Group, China). \\ Comment on: Geserick M, Vogel M, Gausche R, et al. Acceleration of BMI in Early Childhood and Risk of Sustained Obesity. N Engl J Med \\ 2018;379:1303-12.
}

Submitted Dec 04, 2018. Accepted for publication Dec 14, 2018.

doi: 10.21037/atm.2018.12.37

View this article at: http://dx.doi.org/10.21037/atm.2018.12.37

Obesity has become an important public health issue worldwide. A previous study has indicated that agestandardized average body mass index (BMI) increased from $21.7 \mathrm{~kg} / \mathrm{m}^{2}$ in 1975 to $24.2 \mathrm{~kg} / \mathrm{m}^{2}$ in 2014 in men, and from 22.1 to $24.4 \mathrm{~kg} / \mathrm{m}^{2}$ in women worldwide (1). With regard to children, it has been demonstrated that the global agestandardized prevalence of obesity increased from $0.7 \%$ in 1975 to $5.6 \%$ in 2016 in girls (5-19 years old), and from $0.9 \%$ to $7.8 \%$ in boys (5-19 years old) (2). However, few studies have focused on the prevalence of obesity in children under 5 years old and the incremental acceleration in annual BMI in children and adolescents. One of the major strengths of the study being referenced in this editorial was the population-based design with a large cohort encompassing more than 51,000 children across the entire age span from infancy to adolescence. The results from this study show that obesity occurred early in life (3 years of age) and once present, obesity persisted into adolescence in almost $90 \%$. Furthermore, it has been suggested that among obese adolescents, the most rapid weight gain occurs between 2-6 years of age, indicating that BMI acceleration in this period may be useful in predicting obesity during adolescence. These results provided reliable evidences for the epidemiology of childhood obesity, and give new insight into the prevention of obesity in adolescents and adults as well.

Clinicians and researchers hold two points of view on obesity. The mainstream opinion considers obesity a disease. Previous studies have demonstrated that obesity as defined by BMI is related to increased all-cause mortality in the adult population among all racial and ethnic groups (3-6). More recently, Bhaskaran et al. further examined the relationship between BMI and an extensive range of cause-specific mortality outcomes (7). They have found that BMI had J-shaped associations with all-cause, noncommunicable, and communicable disease mortality, with the nadir for all-cause mortality risk at a BMI of $25 \mathrm{~kg} / \mathrm{m}^{2}$ among non-smokers.

An alternative point of view is that obesity alone is not responsible for the development of various medical morbidities (8-11). This viewpoint further suggests that BMI should not be the exclusive indicator used for estimating mortality among the obese population. There are three main reasons for this perspective. Firstly, the causes and mechanisms of obesity are multitudinous. A previous study identified at least 77 loci which have been related to obesity (12). Furthermore, $40-70 \%$ of inter-individual BMI variation has been traced back to genetic factors $(13,14)$. Moreover, the largest genome-wide association studies (GWAS) meta-analysis for BMI identified 97 genome-wide significant (GWS) loci related to BMI, including 56 novel loci (15). The other common instance is that the increasing levels of BMI and fat mass in post-menopause women (16) are result from senility (17) and the decreased level of sex hormone (18). Secondly, the impacts of obesity on different individuals is not identical $(8,9)$. Individuals having high levels of body adiposity who exhibit a cardioprotective phenotype and have a normal metabolic profile are often 
referred to as the "metabolically healthy obese (MHO)" $(19,20)$. Primeau et al. have reported that individuals who are $\mathrm{MHO}$ account for as much as $18 \%$ to $44 \%$ of the adults' population (19). Among children, the prevalence of MHO ranges from $4.2 \%$ to $68 \%$ (21). In addition to this, overweight or obese individuals with impaired metabolic health are defined as the "metabolically unhealthy obesity (MUO)" (22). The increased mortality among the obese population may predominantly be accounted for by MUO individuals. Susceptibility to metabolic or cardiopulmonary dysfunction due to obesity is therefore not mediated by fat content alone, but is dependent on the adipose tissue distribution and the ability of adipose tissue depots to expand (8). Regulation of gene expression, together with epigenetic control of energy balance, determines the status of adiposity of an individual from an early age to later life (8). Thirdly, a concept exists referred to as the 'obesity paradox', which is defined as obesity being protective against mortality in individuals with cardiovascular disease (CVD). Numerous studies have shown that normal weight individuals with CVD have worse survival than do overweight and mildly obesity with the same degree of CVD (10). A study by Flegal et al., found that relative to normal weight, individual with BMI of 30 to $<35$ (grade 1 obesity) was associated with lower mortality, and individual with BMI of 25 to $<30$ (overweight) was associated with significantly lower allcause mortality. This suggests that higher BMI levels may be responsible for driving the increasing mortality among the obese population (23). Indeed, an increasing number of studies have demonstrated the beneficial effects of subcutaneous adipose tissue on metabolism (24). Leptin (25), which is closely related to appetite and glucose metabolism, as well as adiponectin (26), which protects against diabetes, are secreted from subcutaneous fat. More recently, Li et al. have reported that the effect of fibroblast growth factor 21 in enhancing insulin sensitivity depending on specific expansibility of subcutaneous fat (27). Overall, clinicians and researchers therefore should pay attention to overall metabolic status and the cause of obesity rather than BMI alone. Interventions should be focused on fitness-based interventions and physical activity rather than treating all obese individuals with weight-loss driven approaches which may be counterproductive. Further research is needed to explore the MHO phenotype during childhood and to investigate its relationship to the overall metabolic status of patients with pediatric obesity.

With regard to obesity prevention, the paper being referenced in this editorial posited that an excessive weight gain during the interval of 2-6 years of age should be regarded as a crucial period for the prediction of obesity in adolescence. This same age period has also been described as an early sign of ensuing overweight in adulthood (28). These results provided evidence that clinicians should pay more attention to the management of weight gain in patients in this age range. As mentioned earlier, metabolic health should also be considered an important indicator for the assessment of obesity. A recent study, by Cirulli et al., introduced a more accurate approach of categorizing and phenotyping risk of obesity rather than using BMI alone (29). Cirulli et al. used non-targeted metabolomics and whole-genome sequencing to confirm metabolic and genetic signatures of obesity and found that the metabolome captures clinically relevant phenotypes of obesity. They identified 49 metabolites that were associated with BMI, including lipids, amino acids, nucleotides, and peptides, among others. Urate was most significantly associated with BMI among the 49 metabolites. Thereafter, they used ridge regression as well as lasso regression to establish a BMI prediction model which was defined as mBMI on the basis of the metabolome. This model based on the $49 \mathrm{BMI}$-associated metabolites, as well as age and gender explained $43 \%$ of the variation in BMI. In predicting whether participants were obese or normal weight, the model had an area under the curve of 0.922 with $89.1 \%$ specificity and $80.2 \%$ sensitivity. They found that at any given BMI, abnormal metabolomes were associated with different health outcomes; those whose mBMI was greater than their actual BMI were marginally more likely to gain weight and convert to an obese phenotype over the 8-18 years of follow up; and those with healthier metabolomes to have fewer cardiac events. These results are consistent with a favorable long-term health benefit for overweight and obese individuals with a healthy metabolome, and also suggested that the $\mathrm{mBMI}$ can be used as a clinically meaningful metric.

In conclusion, the current trend in obesity management is to combine BMI with metabolic health to categorize obesity and estimate the degree of severity of obesity in children, adolescents, and adults. In addition, more population-based studies that combine of both BMI and metabolic health are needed to explore the all-cause mortality and specific cause mortality in obesity.

This article is worth reading for all providers taking care of patients with obesity, as it provides information for the first time regarding the epidemiology of obesity in children under 5 years old. Additionally, this study gives new insight 
into the value of the BMI acceleration in the period of 2-6 years in predicting obesity in adolescents. We believe this study will give clinicians valuable information to guide their practice treating young children with obesity.

\section{Acknowledgements}

Funding: This work was supported by the National Key R\&D Program of China (No. 2018YFC1314101, 2016 YFC1305600).

\section{Footnote}

Conflicts of Interest: The authors have no conflicts of interest to declare.

\section{References}

1. NCD Risk Factor Collaboration (NCD-RisC). Trends in adult body-mass index in 200 countries from 1975 to 2014: a pooled analysis of 1698 population-based measurement studies with 19.2 million participants. Lancet 2016;387:1377-96.

2. NCD Risk Factor Collaboration (NCD-RisC). Worldwide trends in body-mass index, underweight, overweight, and obesity from 1975 to 2016: a pooled analysis of 2416 population-based measurement studies in 128.9 million children, adolescents, and adults. Lancet 2017;390:2627-42.

3. Di Angelantonio E, Bhupathiraju ShN, Wormser D, et al. Body-mass index and all-cause mortality: individualparticipant-data meta-analysis of 239 prospective studies in four continents. Lancet 2016;388:776-86.

4. Gu D, He J, Duan X, et al. Body weight and mortality among men and women in China. JAMA 2006;295:776-83.

5. Zheng W, McLerran DF, Rolland B, et al. Association between body-mass index and risk of death in more than 1 million Asians. N Engl J Med 2011;364:719-29.

6. Zheng Y, Manson JE, Yuan C, et al. Associations of Weight Gain From Early to Middle Adulthood With Major Health Outcomes Later in Life. JAMA 2017;318:255-69.

7. Bhaskaran K, Dos-Santos-Silva I, Leon DA, et al. Association of BMI with overall and cause-specific mortality: a population-based cohort study of 3.6 million adults in the UK. Lancet Diabetes Endocrinol 2018;6:944-53.

8. Vasan SK, Karpe F. Adipose tissue: Fat, yet fit. Nat Rev Endocrinol 2016;12:375-6.
9. Stefan N, Haring HU, Schulze MB. Metabolically healthy obesity: the low-hanging fruit in obesity treatment? Lancet Diabetes Endocrinol 2018;6:249-58.

10. Kennedy AB, Lavie CJ, Blair SN. Fitness or Fatness: Which Is More Important? JAMA 2018;319:231-2.

11. Barry VW, Baruth M, Beets MW, et al. Fitness vs. fatness on all-cause mortality: a meta-analysis. Prog Cardiovasc Dis 2014;56:382-90.

12. Fall T, Ingelsson E. Genome-wide association studies of obesity and metabolic syndrome. Mol Cell Endocrinol 2014;382:740-57.

13. Visscher PM, Brown MA, McCarthy MI, et al. Five years of GWAS discovery. Am J Hum Genet 2012;90:7-24.

14. Zaitlen N, Kraft P, Patterson N, et al. Using extended genealogy to estimate components of heritability for 23 quantitative and dichotomous traits. PLoS Genet 2013;9:e1003520.

15. Locke AE, Kahali B, Berndt SI, et al. Genetic studies of body mass index yield new insights for obesity biology. Nature 2015;518:197-206.

16. Poehlman ET, Toth MJ, Gardner AW. Changes in energy balance and body composition at menopause: a controlled longitudinal study. Ann Intern Med 1995;123:673-5.

17. Misso ML, Jang C, Adams J, et al. Differential expression of factors involved in fat metabolism with age and the menopause transition. Maturitas 2005;51:299-306.

18. Chen Z, Bassford T, Green SB, et al. Postmenopausal hormone therapy and body composition--a substudy of the estrogen plus progestin trial of the Women's Health Initiative. Am J Clin Nutr 2005;82:651-6.

19. Primeau V, Coderre L, Karelis AD, et al. Characterizing the profile of obese patients who are metabolically healthy. Int J Obes (Lond) 2011;35:971-81.

20. Karelis AD. To be obese--does it matter if you are metabolically healthy? Nat Rev Endocrinol 2011;7:699-700.

21. Farello G, Antenucci A, Stagi S, et al. Metabolically healthy and metabolically unhealthy obese children both have increased carotid intima-media thickness: a case control study. BMC Cardiovasc Disord 2018;18:140.

22. Wildman RP, Muntner P, Reynolds K, et al. The obese without cardiometabolic risk factor clustering and the normal weight with cardiometabolic risk factor clustering: prevalence and correlates of 2 phenotypes among the US population (NHANES 1999-2004). Arch Intern Med 2008;168:1617-24.

23. Flegal KM, Kit BK, Orpana H, et al. Association of allcause mortality with overweight and obesity using standard 
body mass index categories: a systematic review and metaanalysis. JAMA 2013;309:71-82.

24. Tran TT, Yamamoto Y, Gesta S, et al. Beneficial effects of subcutaneous fat transplantation on metabolism. Cell Metab 2008;7:410-20.

25. Lee YH, Magkos F, Mantzoros CS, et al. Effects of leptin and adiponectin on pancreatic beta-cell function. Metabolism 2011;60:1664-72.

26. Kadowaki T, Yamauchi T. Adiponectin and adiponectin receptors. Endocr Rev 2005;26:439-51.

Cite this article as: Zhu B, Gusdon AM, Qu S. Weight or metabolism: which deserve more attention in obesity? Ann Transl Med 2018;6(Suppl 2):S127. doi: 10.21037/atm.2018.12.37
27. Li H, Wu G, Fang Q, et al. Fibroblast growth factor 21 increases insulin sensitivity through specific expansion of subcutaneous fat. Nat Commun 2018;9:272.

28. de Kroon ML, Renders CM, van Wouwe JP, et al. The Terneuzen Birth Cohort: BMI change between 2 and 6 years is most predictive of adult cardiometabolic risk. PLoS One 2010;5:e13966.

29. Cirulli ET, Guo L, Leon Swisher C, et al. Profound Perturbation of the Metabolome in Obesity Is Associated with Health Risk. Cell Metab 2018. [Epub ahead of print]. 